\title{
Brain, mind and limitations of a scientific theory of human consciousness
}

\author{
Alfred Gierer \\ Max Planck Institute for Developmental Biology \\ Spemannstr. 35, D- 72076 Tübingen
}

\section{Summary}

In biological terms, human consciousness appears as a feature associated with the functioning of the human brain. The corresponding activities of the neural network occur strictly in accord with physical laws; however, this fact does not necessarily imply that there can be a comprehensive scientific theory of consciousness, despite all the progress in neurobiology, neuropsychology and neurocomputation. Predictions of the extent to which such a theory may become possible vary widely in the scientific community. There are basic reasons - not only practical but also epistemological - why the brain-mind relation may never be fully "decodable" by general finite procedures. In particular self-referential features of consciousness, such as self-representations involved in strategic thought and dispositions, may not be resolvable in all their essential aspects by brain analysis. Assuming that such limitations exist, objective analysis by the methods of natural science cannot, in principle, fully encompass subjective, mental experience.

Key words: brain / mind / consciousness / human / decodability / decision theory / Gödel / Heisenberg / Frayn

\section{Introduction}

"It is a very difficult task, in any respect, to form a solid opinion on the mind (psyche, soul) ... It seems as though everything the soul experiences occurs only in association with a body... joy, as well as love and hate; in all of these cases something happens in the body as well... If this is the case, then these properties possess something material even in their very essence... And that is, after all, the reason why the physicist is responsible for the investigation of the soul."

These sentences are 2300 years old, and are found in the introduction to the first systematic discussion of the mind-body problem, namely Aristotle's 'De anima'. At their core they are still valid today. The mind-body problem remains as fascinating as ever; it is difficult to solve; and it revolves mainly around the relationship between corporeal and mental processes, which are perceived in very different ways and expressed in terms of different concepts. While we can objectively observe and measure bodily processes such as speaking and blushing, and modern techniques allow us to detect brain activities as well, within our conscious mind we are directly exposed to our own state which manifests itself in the form of feelings, thoughts, intentions, memories, wishes, fears, and hopes, often independent of the senses and almost always without knowing the simultaneous physical processes in the brain. Conscious experience is 
thus not primarily given in spatio-temporal terms and can therefore not be directly analyzed in terms of natural science. Yet it can be expressed and communicated through speech and thus be subject to scientific discussion. Some expressions of mental states, such as laughter and crying are innate while others, particularly those associated with speech, are dependent on learning and transmitted through culture. Using a thesaurus one is impressed by the sheer number of expressions involving the 'mental' domain. About one half of the words in 'Roget's Pocket Thesaurus' can be found under such headings as 'intellectual faculties', 'voluntary powers', 'sentient and moral powers'. Even if we reduce this number by applying very strict criteria, there remain thousands of expressions belonging to the mental domain. Combining them allows intersubjective communication of an enormous number of different mental states and processes.

Now what is the relationship between mental and physical aspects? In discussing this basic question, some remarks on the use of the term "physical" are appropriate. In 1958, Feigl ${ }^{1}$ wrote his stimulating article "The Mental and the Physical" that has contributed much to reviving scientific interest in the brain-mind problem. In order to allow for the general distinction from the mental, "physical" is to be understood as encompassing the entire hierarchy of objects of natural science, such as molecules, cells and organs in biology. All these systems and subsystems are characterized by phenomena, concepts and methods specific for each level, and yet a generalisation of the meaning of physical, beyond the realm of what is called pure physics in contemporary academic curricula, is adequate because all objects of natural science follow the same fundamental laws of physics, in particular with respect to the dynamic interactions of their components. This fully applies to the brain, and it is this feature that is highly relevant to our topic.

By now, the neurosciences have led to rapidly increasing insights into correlations between sensory perception, language and emotions with increased neural activity in specific areas of the brain. Some functions, such as those involving long-term memory and thought, seem to be distributed across wide areas, but also modu- larly. Noninvasive monitoring allows us to discover more and more relations between brain activities and their locations. By now, there are tens of thousands of studies on patterns of activity of the human brain: studies on attention, on various types of mental processes including calculations, even on self-representation, representation of others (often called "theory of mind"), and empathy.

In the chronological organization of processes and, in particular, in the planning of future behavior, the prefrontal cortex plays a special role. These functions require the emotive evaluation of desirable and undesirable situations and scenarios. Correspondingly, the cortex is extensively and subtly interconnected with centers involved in memory and feelings in areas of the limbic system.

The monitoring of brain activities in relation to conscious processes represents only one approach, albeit an impressive one, in the investigation into the neurobiological principles behind the conscious mind. It is supplemented by other methods addressing neural activity, connectivity and function. In addition, there is an entire spectrum of research fields - such as psychophysical methods, comparative investigations on other primates, the study of models of neural networks and the corresponding computer-based theoretical research - that may contribute to the understanding of higher brain functions, such as pattern recognition and language, learning and memory, voluntary movement, chronological organization of actions and many other abilities. All in all we are increasingly able to understand many relationships between processes in the brain and processes in the mind. Most activities of the human brain are unconscious and routine; conscious processes are heavily influenced by unconscious pre-conditions, such as past experience and emotions. Consciousness is mediated by the cerebral cortex, and it is activated particularly by situations that are novel or that involve difficult planning or decisions. Models for the underlying integrative features of large neural networks or even the entire cortex are particularly interesting in this context. Examples are models of "binding" distributed activities as analyzed by Crick and $\mathrm{Koch}^{2}$, and the concept of Dehaene, Kerszberg and Changeux ${ }^{3}$ postulating a "global workspace" for dealing with "effortful 
cognitive tasks". The main topic of this article is to what extent neuroscience can be expected to eventually explain or resolve the brain - mind - relationship.

\section{The spectrum of opinions on the brain-mind- relation is wide}

While there is a continuous increase of knowledge on specific neurobiological aspects of distinct mental processes, there are some main basically different lines of general philosophical thoughts and attitudes concerning brain, mind and consciousness that are rather persistent.

The progress made in brain research has been so impressive that many neurobiologists, including some of the most creative scientists in the field, tend to assume that there will be an asymptotic approach to a complete understanding of the $\operatorname{mind}^{2,4,5}$. This attitude, whether implicit or explicit, is not necessarily related to specific philosophical concepts which are often viewed as unnecessary. Consciousness appears as a property of systems of nerve cells in the brain, and scientific progress has led to the understanding of systems features even in very difficult cases. For example, supraconductivity is the property of systems of certain metal atoms at low temperatures. After all we have learned how to understand supraconduction on the basis of physical laws and processes why should this not be possible for consciousness as a system's property of certain neural networks? But such comparisons are not completely apposite. Supraconduction is objectively defined - the electrical resistance is zero - consciousness is not. Of course one can nevertheless try to define consciousness in formal terms. If human consciousness is to be encompassed, one quickly agrees that its self-referential property is a central feature. Integration in time - past and future - and symbolic thought are also essential. But these features are not yet enough for a comprehensive definition of the mental aspects; one could build self-referential properties, when they have been formally defined, into a pocket computer, but we would still not consider it conscious. Consciousness is primarily accessible through self-awareness and through the communication of the awareness of others; it is doubtful whether in principle a complete formal or objective definition can be made. For these reasons, it is also doubtful whether the evolution of human consciousness can be fully and objectively explained in scientific terms.

Another position which one finds to be represented in almost every symposium on the psychophysical relationship is the thesis that we are dealing with an artificial, illusory problem ${ }^{6}$. In this view, all one needs to do is to explain the terminology properly, to clearly define what one means - which are for example, the semantic and epistemological problems in grasping the minds of others, and in describing mental states of oneself - and the mind-body problem will disappear. Is it just an artificial product of philosophy since Descartes, if not Platon? In a very informative chapter on the history of the mind-body-problem, going back to prehistoric times, Popper ${ }^{7}$ argued rather convincingly against this. In the tenth canto of Homer's Odyssey, Circe transforms men into swine. After this unfortunate action, the condition of Odysseus' companions is described as follows: 'They had the heads, voices and bodies of swine. Only their minds remained as before.' Body and mind are seen as distinct, but interrelated: the companions understood their frightful situation. Generally, the "conscious self" appears as a universal experience of mankind. According to modern biology and psychology, conscious experience is closely related to physical processes in the brain, and this relationship cannot be dismissed as something not belonging to the realm of scientific inquiry.

A further line of thought in the spectrum of opinion holds that understanding the mind-body relationship is not possible in the current state of physics, but a future expanded physics could fully resolve the problem. The world of atoms was not accessible to the physics of the beginning of the 20th century, but the new discipline of quantum mechanics - and the underlying conceptual changes and expansions of the basic laws of physics - rendered atoms and molecules understandable in physical terms. Why should an expanded form of current physics not be able to explain consciousness? This position is representented, for example, by Penrose ${ }^{8}$. Although it is widely criticized ${ }^{9}$, and is very much a minority 
opinion, it must be taken seriously; but the likelihood of this happening is something I would bet against.

And yet, contemporary discussions on the meaning and the philosophical interpretation of quantum theory which is fundamental to so much of physics, and virtually to all of chemistry, are not irrelevant and may still be stimulating for thoughts on the brain-mind-relation. Though direct conclusions from quantum computation on consciousness, or from quantum uncertainty on free will, are inadequate, it is obvious that the material and mental aspects of modern physics are rather remote from the full-blooded mechanisticmaterialistic determinism of nineteenth century physics. We should not rely too much, in our intuitions, on these outdated notions when it comes to general implications of brain research for human self-understanding.

In the following, I would like to explicate a position that appears as the most reasonable one to me: there are principle reasons standing in the way of a complete decoding of the relationship between the brain and mind. This position is supported by more than a few, but is probably not a majority opinion in the scientific community. It is based on two premises: stringent physicalism - physics is valid everywhere, including the human brain - and epistemological scepticism. Neurobiology will take us further in our understanding, as many believed a short time ago. But there will be unsurpassable limits, particularly where self-referential processes in the brain are concerned ${ }^{10,11}$.

\section{Range and limits of a scientific understanding of human consciousness}

The neural network of the human brain consists of well over ten billion nerve cells, connected by hundreds of thousands of kilometers of neural fibers. They make up thousands of billions of synaptic connections between nerve cells. A basic function of the brain is storing and processing information. Now we know that every function of information processing that one can model in formal, mathematical terms can, in principle, be executed by a computer on the basis of physical interactions of its constituents. Because the nerve cell's capacity as a building block for information processing is greater, not smaller than that of the digital yes/no switch of computers, one expects all the formally representable functions of information processing of the human brain to be based on physical chemical processes in the nervous system. This argument indicates that a scientific explanation is possible in principle but it is not the explanation in itself, which can only be achieved by neurobiology. And then there is the important question to which extent features and functions of the brain can actually be described formally as information processing. What can be formally represented can be seen in the research into artificial intelligence, and the list is impressive: object recognition, conceptual abstraction, memory, planning and the comparison of different strategies for future behavior are all on this list - in other words, much of what one considers to be the higher capabilities of the brain.

How far will such investigations take us? Does it depend solely on our efforts, or are there limits in principle - limits not just concerning complex details, but also limits to intrinsic, interesting, and central features of consciousness? When mental states are unambigously linked to physicalchemical states of the nervous system, and these follow the laws of physics, it is tempting to assume that a comprehensive scientific theory of the brain-mind relationship should be possible. However, this assumption is subject to criticism upon closer analysis.

Let us first consider, for comparison, knowledge about range and limits of physics and mathematics. In these fields, there are many questions that become more answerable, the more effort we put in. For instance, energy states of stable material systems can be determined very exactly, depending on the effort involved; in this way, for instance, we understand chemical bonds very well. But there are also questions for which there are no definitive answers no matter how much effort you put in. Predicting, precisely, individual events on the atomic level is, in principle, not possible regardless of the efforts involved in measurement and calculation, as we know from the famous "uncertainty principle" of quantum physics that Heisenberg discovered. Quantum physics has 
included these limits in its basic equations from the outset: uncertainty, i.e. the limitation of possible knowledge, is itself a law of nature. Uncertainty is not just limited to the atomic level, but can also apply on a large scale whenever individual events on the atomic level are amplified to lead to macroscopic changes. Sexual reproduction, for instance, is dependent on processes in individual molecules of the genetic material DNA, on the random breaking and combining of chromosomal nucleic acid, and these processes are subject to quantum uncertainty.

There is another epistemological limit to our knowledge that has been uncovered by mathematical decision theory. In the 1920 s it was still considered a goal of mathematics to arrive at a formal system of mathematics and logic, allowing for the proof of its internal consistency - the proof that contradicting statements cannot possibly arise within the system. It was Kurt Gödel's great discovery in $1931^{12}$ that this is impossible for stringent mathematical reasons. Logical systems, except very primitive ones, do not allow proving their consistency by using their own means. Within every such system of formal thought and calculation, there are questions that cannot be decided in principle.

It is remarkable that physical uncertainty and mathematical undecidability, though applying to different domains of knowledge, are logically related: in both cases, problems of self-reference are involved, the effects of measurements on the states to be measured in physics, and the logic of logic in mathematics. I would like to name reasons why there could be limitations for a comprehensive scientific theory of human consciousness ${ }^{10,13,14}$, reasons also linked to features and processes involving self-reference.

\section{The brain-mind relation may not be fully de- codable in principle}

A satisfactory theory of the brain-mind relationship would have to encompass a general and reliable method for deducing mental states and activities from data on physical brain states, a procedure that may be described as "decoding" physical brain states with respect to corresponding men- tal states. This is already possible to some extent, as in cases in which certain mental states and processes can be inferred from neuroimaging of brain areas by activity-dependent nuclear magnetic resonance; but we are now asking for intrinsic limitations. Decoding is not sufficient, per se, for explanation, but unsurmountable limitations of decodability may reveal epistemologically relevant limits of scientific explanations of mind.

Let us select, as an example for states involving consciousness, general behavioral dispositions for the future, that is, intentions and inclinations of an individual for various patterns of behavior depending on various scenarios. The capability of generating very general and far-reaching strategic behavioral dispositions is characteristic of highly integrated functions of the human brain; their discussion may lead us further towards an understanding of the possible range and limits of a scientific theory of human consciousness than studies on the role of consciousness in simple voluntary movements ${ }^{15}$. Strategic behavioral dispositions are stored in our brain and are partially accessible to consciousness. Let us perform a thought experiment: let us suppose that we can simulate states and processes of the brain by a correspondingly constructed and programmed computer. In principle, we could calculate, over time, what would happen to a given initial brain state when exposed to certain exterior conditions, and which behavioral responses would result. One could now argue that we could thus test all possible exterior conditions of the future, one after the other, with the final goal of determining the general behavioral dispositions corresponding to the initial state of the brain, but valid for different scenarios in an open future - and in this way to decode the present brain state with respect to conscious states objectively and exhaustively, at least as far as they are related to behavioral dispositions.

But, on second thought, we realize that this would not work; a procedure of this sort proves to be impossible to perform if we consider the finiteness of the world and take it seriously in epistemological terms: the intrinsic finiteness of the world also limits the decidability of problems. Even a computer made up of the mass of the entire universe, running 15 billion years - the age of the 
universe -, would still only be able to execute a finite number of operations - a very liberal upper limit would be $10^{120}$. Such an upper limit of some $10^{120}$ elementary steps of intra-universe information processing is not an arbitrary figure without epistemological status; it is given by the number of relatively stable particles (some $10^{80}$ nucleons) and the maximal number of operations per particle (about $10^{40}$ ) consistent with the stability of the particle and the age of the universe. Estimates of these very large numbers depend to some extent on cosmological models and data, but they are essentially based on elementary constants of physics, Planck's constant, the gravitation constant, the mass of the nucleon, and the speed of light ${ }^{16}$. It is for this reason that, in my view, the corresponding limitations are fundamental, supporting a finitistic epistemology.

Numbers of this huge magnitude do occur even in everyday problems as the number of possibilities. The number of possible letters with various contents, even when only a few pages long, is much larger. The same holds true for the number of possible future physical states that a particular behavioral disposition may apply to. The number of possible different behavioral dispositions is also so large that they certainly could not be checked by processing them one after the other in a finite decision-making process to find out which of these dispositions actually correspond to a given physical state of the brain. It follows that according to finitistic epistemology, mental states are not automatically included in a physical description of the state of the brain; there is no algorithm for their comprehensive deduction.

Of course, it is possible in any field of science to discover many general relations, rules and laws applying to widest domains not limited by cosmological scales, through clever observations, experiments, and theoretical, mathematical thoughts. This also holds true for the mind-brainrelationship; but there will presumably be no general process for discovering every relationship of general validity. It is more likely that some essential aspects of the body-mind-relationship are not automatically decodable in a finite number of steps.

As for the aspects of consciousness that a sci- entific theory may not be able to fully encompass, only more or less educated guesses are possible. We can find hints by drawing analogies to the aforementioned mathematical decision theory: the standard example for principally undecidable questions, the proof of the internal consistency of mathematical-logical systems by their own means, possesses a pronouncedly self-referential character. Analogous to this, the characteristic properties of consciousness, like the generation of behavioral dispositions, are also self-referential. We appear in our own memories, fears and hopes, desires and plans - as we are, or as we believe ourselves to be, or as we wish to be seen by others, as we want or do not want ourselves to become and as we see our past, and our future possibilities. Behavioral dispositions are influenced by these 'self-images', which of course do not represent concrete spatial conceptions, but are rather abstract representations of features of the individual in his or her own brain. Self-images are often contradictory and can never be complete because no physically existing entity can contain a complete duplicate of itself. Selfimages change in the course of time and alternate within conscious experience. They interact with one another and feed back on themselves. Perhaps these multiple self-images belong to the aspects of consciousness that cannot be determined fully by analysis of the physical state of the brain.

To sum these considerations up, it is not a stringent consequence of the applicability of physics to the brain and the unique correspondence of mental states to physical states of the brain that all behavioral dispositions will be deducible from the physical state of the brain in a finitistic process. We have more reason to believe that there are limits to the decodability of brain states with respect to mental states. According to everything that we know, the brain follows the same physical laws as do machines; but a machine that we were capable of understanding could not do everything like a human, and a machine that could do everything like a human would be impossible for us to fully understand. If we know the mental state of a human, expressed by means of language and gestures, we may know more than would be possible to know through a purely physical analysis of her or his brain, however elaborate that analysis may be. 
At the beginning of the 20th century a commonly held belief was that mathematical mechanics at least in principle would be capable of calculating and predicting all physical processes and states - this becomes asymptotically more and more attainable, the more effort we put into it. Since around 1927 - namely since the advent of quantum physics - we know that this is not true. Correspondingly, most mathematicians around 1900 believed in the asymptotic solvability of all logical questions that could be reasonably formulated - including the logical validation of logic - and since Goedel's work of 1931, we have known that this is not true either. Nowadays, in the beginning of the 21 st century, many neurobiologists and researchers into consciousness believe in the asymptotic solvability of the brain-mind relationship; they hold that our knowledge depends essentially on our efforts, which corresponds to the mainstream position in mathematics and physics early in the 20th century. Will this situation look the same in 2030 or in 2100 ? I am one of those who think that is unlikely - one of those who believes that there are basic questions in this field that are, in principle, irresolvable.

\section{Philosophical implications: Some facets of hu- man cognition and human nature}

Of particular interest in this context are the selfreflexive aspects of mental processes that play such a great role in artistic expression as well as in the scientific debates on consciousness. As mentioned before, this feature has close ties to the selflimits of mathematical and scientific thought discovered in the last century; to physical uncertainty and mathematical undecidability, to Heisenberg's law and Gödel's theorem. Both reflect the intricacies of feedback by features and processes, be they physical or mental, onto their own perequisites. Both have something to do with the fact that we are in our physical selves and in our thoughts an inseparable part of the finite world with finite ressources for observation, analysis and computation. Understanding the range and limits of scientific explanations of consciousness has analogous logical facets. Objective physical and neurocomputational analysis of the brain is finite in princi- ple and may reach its limits when self-referential processes and features are involved. Psychological analysis interferes, in effect, with the mental states to be analysed, dealing ultimately with consciousness of consciousness.

Self-reflexive human thought and knowledge are generally sensitive to self-contradiction and are incomplete - but we are increasingly able to know what we can know and what not, and why. The basic limitations of knowledge are concerned with the relationship between the order of nature and human cognition, and they are linked, in this way, with fundamental questions of man's image of himself and the universe. It is such knowledge about the limits of knowledge that shows us why scientific knowledge, despite of its unambiguous contents with respect to spatio-temporal processes and laws, is and remains ambiguous on the metatheoretical level. In contrast to many ideas that existed in the nineteenth century, modern science is capable of being, and needs to be, interpreted on the philosophical, cultural and religious levels, and is consistent with different, though of course not all, such interpretations. The ancient Greek philosophers gave us building blocks for possible interpretations - logos, number, idea, spirit, element. Interpretation itself, however, is the task of the present, a task for science, art and the humanities, even if the languages spoken in different sections of human culture are often quite different from one another.

It is not uninteresting to see how we, in real life, come to terms with more or less hidden inconsistencies and ambiguities of human thought. There is a category of jokes that one could name "metatheoretical". Example: Nasredin Hodscha becomes the village judge without knowing much about the job. A participant in a dispute presents his case and Hodscha declares, "You are right". The opponent presents his point of view, and Nasredin Hodscha says, "You are right", which provokes an angry outburst from the complainant. "You said I was right, and now you're telling him that he's right. We can't both be right." And Nasredin Hodschas final judgment before he leaves the courtroom: "You are right". The joke is clearly based on the last statement, the one about being right about being right; it is self-referential 
and therefore subject to contradiction. We are predisposed to handle such contradictions well. If it is serious, we become alert, otherwise we laugh about it. But the contradiction remains - why not?

An intriguing social aspect of our understanding of consciousness is its relation to cognitionbased empathy, the capability of perceiving mental states of others. E. T. A. Hoffmann's story of the Sandman (more widely known from Offenbach's opera "The Tales of Hoffmann") may illustrate this point: a physicist constructs a lifelike doll with rather lifelike behavior. The physicist's assistant falls in love with the doll, but falls out of love just as soon as he recognizes that he was in love with a machine. In order to feel real empathy and to recognize another consciousness as human, objective criteria do not suffice; we must know or accept that the other entity involved is a conscious human being like us. No one would be charged with murder if he or she destroy the doll, no matter how similar it looks and acts as a human being.

Finally, the problem of consciousness is tied closely to one of the most difficult questions surrounding our understanding of ourselves: the question of free will. Conscious thought is often involved when evaluation of a situation reveals different possible behavioral pathways of comparable emotional desirability. Naturally, the question whether and to which extent we may consider the pathways actually taken as determined will not be resolved solely by insights into possible limits of a theory of the brain-mind relationship; but they make a small contribution. They say namely that there may be principal limits to grasping the consciousness of others. The will of an other, despite being tied closely to processes in his or her brain, cannot be completely decoded by an outsider, and therefore not objectively understandable. Outsiders cannot claim to make certain statements about our own motives if we do not voluntarily share them. Luckily there are limits to intruding into the consciousness of others, and there is unfortunately often too little modesty and reservation when judging the motivations of others. In fact, complete mind reading is beyond human capabilities.

\section{Limits to understanding of self - Example: Heisenberg and Bohr in "Copenhagen"}

Do we at least know enough about ourselves, do our own conscious experiences, emotions and thoughts lead to actual self-understanding? This is an old topic about which psychology, literature and art can contribute more than neurobiology. I just want to confirm that even from a point of view of natural sciences, the answer can be 'no' - that we gain knowledge of ourselves only partially, and often only through our own actions. Through our own acts we become different from our previous selves. Often, we learn about our motives only in hindsight, and then only in a limited way.

At this point I would like to mention a most interesting play on these subjects. The author is Michael Frayn and the name of the play is "Copenhagen". There, around 1927, Heisenberg and Bohr discovered and explained quantum indeterminacy, the principle that is fundamental to modern physics. This achievement is often called the "Copenhagen interpretation" of quantum theory. Fourteen years later, in 1941, Heisenberg, by then one of the heads of the German uranium project during the war, visited his elder teacher and friend Bohr in Nazi-occupied Denmark, in order to discuss the topic of 'atomic bombs'. The visit was a disaster. Bohr never wanted to talk about it again and Heisenberg suffered for the rest of his life from various contradictory, mostly negative versions of what he said, thought and planned at the time. In the play, they are both dead and meet in Heaven along with Margareth, Bohr's wife, who plays the most critical role, with respect to Heisenberg's behavior. They want to find out what really happened in 1941, what was thought, done, said and wanted. It is still not a complete success, but the reciprocal understanding and mood both improve. That is due to the insight into the logical relationship between the limits of self-knowledge and the limits of any knowledge about reality, as shown by the uncertainty of quantum physics that the protagonists Bohr and Heisenberg themselves discovered. In the appendix to his play the author describes briefly and quite well what one really knows about the events of that time. Then, as a writer, he summarizes his ideas on consciousness which are sim- 
ilar to my position as a scientist, and I would like to conclude with the corresponding statements by Frayn $^{17}$ :

"What the uncertainty of thoughts does have in common with the uncertainty of particles is that the difficulty is not just a practical one, but a systematic limitation which cannot even in theory be circumvented. It is patently not resolved by the efforts of psychologists and psychoanalysts, and it will not be resolved by neurologists either, even when everything is known about the structure and workings of our brain..."

\section{References}

1. Feigl H. 1958. The Mental and the Physical. Minnesota Studies II. Minnesota: University of Minnesota Press.

2. Crick F, Koch C. 1998. Consciousness and neuroscience. Cerebral Cortex 8: 97-107.

3. Dehaene S, Kerszberg M, Changeux J.P. 1998. A neuronal model of a global workspace in effortful cognitive tasks. Proc Natl Acad Sci USA 92: 14529-14534.

4. Edelman GE. 2003. Naturalizing consciousness. Proc Natl Acad Sci USA 100: 5520-5524.

5. Edelman GE. 2006. The embodiment of mind. Daedalus Summer 2006: 23-32

6. Ryle G. 1949. The Concept of Mind. London: Hutchinson.

7. Popper KR. 1977. Historical comments on the mind-body problem. In Popper KR, Eccles JC; The Self and its Brain. Berlin: Springer Berlin. p 148-208.

8. Penrose R. 1996. Shadows of the Mind: A Search for the Missing Science of Consciousness. Oxford: Oxford University Press.

9. Litt A, Eliasmith C, Kroon FW, Weinstein SW, Thagard P. 2006. Is the brain a quantum computer? Cognitive Science 30: 593603.

10. Gierer A. 1983. Relation between neurophysiological and mental states: possible limits of decodability. Naturwissenschaften 70: 282-287.

11. Chalmers DJ. 1995. The Puzzle of Conscious Experience. Scientific American1995: 62-68.

12. Gödel K. 1931. Über formal unentscheidbare Sätze der Principia Mathematica und verwandter Systeme. Monatshefte für Mathematik und Physik 38: 173-198.

13. Gierer, A. 1970. The physical foundations of biology and the problems of psychophysics. Ratio XII: 47-64.

14. Gierer A. 2005. Biologie, Menschenbild und die knappe Ressource Gemeinsinn. Würzburg: Koenigshausen \& Neumann.

15. Libet B, Gleason CA, Wright EW, Pearl DK. 1983. Time of conscious intention to act in relation to onset of cerebral activity (readiness potential) - the unconscious initiation of freely voluntary act. Brain 106: 623-642.

16. Barrow JD. 1990. The mysterious lore of large numbers. In Bertotti B,Balbinot R, Bergia S,Messina A. ed; Modern Cosmology in Retrospect. Cambridge: Cambridge University Press. p 67-93.

17. Frayn, M. 1998. Copenhagen. London: Methuen Drama. p 101. 